\title{
Fresnelets: New Multiresolution Wavelet Bases for Digital Holography
}

\author{
Michael Liebling, Student Member, IEEE, Thierry Blu, Member, IEEE, and Michael Unser, Fellow, IEEE
}

\begin{abstract}
We propose a construction of new wavelet-like bases that are well suited for the reconstruction and processing of optically generated Fresnel holograms recorded on CCD-arrays. The starting point is a wavelet basis of $L_{2}$ to which we apply a unitary Fresnel transform. The transformed basis functions are shift-invariant on a level-by-level basis but their multiresolution properties are governed by the special form that the dilation operator takes in the Fresnel domain. We derive a Heisenberg-like uncertainty relation that relates the localization of Fresnelets with that of their associated wavelet basis. According to this criterion, the optimal functions for digital hologram processing turn out to be Gabor functions, bringing together two separate aspects of the holography inventor's work.

We give the explicit expression of orthogonal and semi-orthogonal Fresnelet bases corresponding to polynomial spline wavelets. This special choice of Fresnelets is motivated by their near-optimal localization properties and their approximation characteristics. We then present an efficient multiresolution Fresnel transform algorithm, the Fresnelet transform. This algorithm allows for the reconstruction (backpropagation) of complex scalar waves at several user-defined, wavelength-independent resolutions. Furthermore, when reconstructing numerical holograms, the subband decomposition of the Fresnelet transform naturally separates the image to reconstruct from the unwanted zero-order and twin image terms. This greatly facilitates their suppression. We show results of experiments carried out on both synthetic (simulated) data sets as well as on digitally acquired holograms.
\end{abstract}

Index Terms-B-splines, digital holography, Fresnel transform, Fresnelet transform, Fresnelets, wavelets.

\section{INTRODUCTION}

D IGITAL holography [1]-[4] is an imaging method in which a hologram [5] is recorded with a CCD-camera and reconstructed numerically. The hologram results from the interference between the wave reflected or transmitted by the object to be imaged and a reference wave. One arrangement that is often used is to record the distribution of intensity in the hologram plane at the output of a Michelson interferometer. The digital reconstruction of the complex wave (amplitude and phase) near the object is based on the Fresnel transform, an approximation of the diffraction integral [6].

Digital holography's applications are numerous. It has been used notably to image biological samples [7]. As the range of applications gets broader, demands toward better image quality increases. Suppression of noise, higher resolution of the recon-

Manuscript received December 17, 2001; revised October 1, 2002. The associate editor coordinating the review of this manuscript and approving it for publication was Prof. Pierre Moulin.

The authors are with the Biomedical Imaging Group, STI, BIO-E, Swiss Federal Institute of Technology, Lausanne (EPFL), CH-1015 Lausanne, Switzerland (e-mail: michael.liebling@epfl.ch).

Digital Object Identifier 10.1109/TIP.2002.806243 structed images, precise parameter adjustment and faster, more robust algorithms are the essential issues.

Since it is in essence a lensless process, digital holography tends to spread out sharp details like object edges over the entire image plane. Therefore, standard wavelets, which are typically designed to process piecewise smooth signals, will give poor results when applied directly to the hologram. We present a new family of wavelet bases that is tailor-made for digital holography.

While analytical solutions to the diffraction problem can be given in terms of Gauss-Hermite functions [6], those do not satisfy the completeness requirements of wavelet theory [8] and are therefore of limited use for digital processing. This motivates us to come up with basis functions that are well-suited for the problem at hand. The approach that we are proposing here is to apply a Fresnel transform to a wavelet basis of $L_{2}$ to simulate the propagation in the hologram formation process and build an adapted wavelet basis.

We have chosen to concentrate on B-spline bases for the following reasons.

- The B-splines have excellent approximation characteristics (in some asymptotic sense, they are $\pi$ times better than Daubechies wavelets [9]).

- The B-splines are the only scaling functions that have an analytical form in both time and frequency domains; hence, there is at least some hope that we can derive their Fresnel transforms and associated wavelets explicitly.

- The B-splines are nearly Gaussians and their associated wavelets very close to Gabor functions (modulated Gaussians) [10]. This property will turn out to be crucial because we will show that these functions are well localized with respect to the holographic process.

The paper is organized as follows. In Section II, we define the unitary Fresnel transform in one and two dimensions. In Section III we review several of its key properties that are needed in order to define the new bases. We also investigate the spatial localization properties of the Fresnel transform and derive a Heisenberg-like uncertainty relation. In Section IV, we define the Fresnelet bases. We briefly review B-splines and their associated wavelet bases and show how to construct the corresponding Fresnelet bases. We derive an explicit closed-form expression for orthogonal and semi-orthogonal Fresnelet bases corresponding to polynomial spline wavelets. We also discuss their properties including their spatial localization and multiresolution structure. In Section V, we show how to implement our multiresolution Fresnel transform. Finally, in Section VI, we apply our method to the reconstruction of holograms using both simulated and real-world data. 
In the sequel, we use the following definition of the Fourier Transform $\hat{f}(\nu)$ of a function $f(x)$ :

$$
\begin{aligned}
& \hat{f}(\nu)=\int_{-\infty}^{\infty} f(x) e^{-2 i \pi x \nu} d x \\
& f(x)=\int_{-\infty}^{\infty} \hat{f}(\nu) e^{2 i \pi \nu x} d \nu .
\end{aligned}
$$

With this definition $\|f\|=\|\hat{f}\|$.

\section{FRESNEL TRANSFORM}

\section{A. Definition}

We define the unitary Fresnel transform with parameter $\tau \in$ $\mathbb{R}_{+}^{*}$ of a function $f \in L_{2}(\mathbb{R})$ as the convolution integral

$$
\tilde{f}_{\tau}(x)=\left(f * k_{\tau}\right)(x) \text { with } k_{\tau}(x)=\frac{1}{\tau} e^{i \pi(x / \tau)^{2}}
$$

which is well defined in the $L_{2}$ sense. Our convention throughout this paper will be to denote the Fresnel transform with parameter $\tau$ of a function using the tilde and the associated index $\tau$.

The frequency response of the Fresnel operator is

$$
\hat{k}_{\tau}(\nu)=e^{i \pi / 4} e^{-i \pi(\tau \nu)^{2}}
$$

with the property that $\left|\hat{k}_{\tau}(\nu)\right|=1, \forall \nu \in \mathbb{R}$. As the transform is unitary, we get a Parseval equality

$$
\forall f, g \in L_{2}(\mathbb{R}) \quad\langle f, g\rangle=\left\langle\tilde{f}_{\tau}, \tilde{g}_{\tau}\right\rangle
$$

and for $f=g$ a Plancherel equality

$$
\forall f \in L_{2}(\mathbb{R}) \quad\|f\|=\left\|\tilde{f}_{\tau}\right\| .
$$

Therefore, we have that $\tilde{f}_{\tau} \in L_{2}(\mathbb{R})$.

The inverse transform in the space domain is given by

$f(x)=\left(\tilde{f}_{\tau} * k_{\tau}^{-1}\right)(x)$ with $k_{\tau}^{-1}(x)=k_{\tau}^{*}(x)=\frac{1}{\tau} e^{-i \pi(x / \tau)^{2}}$.

It is simply derived by conjugating the operator in the Fourier domain

$$
\hat{k}_{\tau}^{-1}(\nu)=e^{-i(\pi / 4)} e^{i \pi(\tau \nu)^{2}}=\hat{k}_{\tau}^{*}(\nu) .
$$

\section{B. Example: Gaussian Function}

The Fresnel transform of the Gaussian function

$$
g(x)=e^{-\pi(x / \sigma)^{2}}
$$

is again a Gaussian, modulated by a chirp function

$$
\tilde{g}_{\tau}(x)=a e^{-\pi\left(x / \sigma^{\prime}\right)^{2}} e^{i \pi\left(x / \tau^{\prime}\right)^{2}}
$$

where $a=e^{i(\pi / 4)}\left(\sigma / \sqrt{\sigma^{2}+i \tau^{2}}\right)$ is the complex amplitude, $\sigma^{\prime 2}=\left(\sigma^{4}+\tau^{4}\right) / \sigma^{2}$ is the new variance and $\tau^{\prime 2}=$ $\left(\sigma^{4}+\tau^{4}\right) / \tau^{2}$ is the chirp parameter. As the parameter $\tau$ increases, the variance and therefore the spatial spreading of the transformed function increases as well. This aspect of the Fresnel transform is further investigated in Section III-E.

\section{Two-Dimensional Fresnel Transform}

We define the unitary two-dimensional (2-D) Fresnel transform of parameter $\tau \in \mathbb{R}_{+}^{*}$ of a function $f \in L_{2}\left(\mathbb{R}^{2}\right)$ as the 2-D convolution integral

$$
\tilde{f}_{\tau}(\vec{x})=\tilde{f}_{\tau}(x, y)=\left(f * K_{\tau}\right)(\vec{x})
$$

where the kernel is

$$
K_{\tau}(\vec{x})=\frac{1}{\tau^{2}} e^{i \pi(\|\vec{x}\| / \tau)^{2}} .
$$

A key property is that it is separable

$$
K_{\tau}(\vec{x})=\frac{1}{\tau^{2}} e^{i \pi(\|\vec{x}\| / \tau)^{2}}=k_{\tau}(x) k_{\tau}(y) .
$$

Thus, we will be able to perform most of our mathematical analysis in one dimension and simply extend the results to two dimensions by using separable basis functions.

The two-dimensional unitary Fresnel transform is linked to the diffraction problem in the following manner. Consider a complex wave traveling in the $z$-direction. Denote by $\psi(x, y)$ the complex amplitude of the wave at distance 0 and by $\Psi(x, y)$ the diffracted wave at a distance $d$. If the requirements for the Fresnel approximation are fulfilled, we have that [6]

$$
\begin{aligned}
\Psi(x, y) & =\frac{e^{i k d}}{i \lambda d} \iint_{\|} \psi(\xi, \eta) e^{(i \pi) /(\lambda d)\left((\xi-x)^{2}+(\eta-y)^{2}\right)} d \xi d \eta \\
& =-i e^{i k d} \tilde{\psi}_{\sqrt{\lambda d}}(x, y)
\end{aligned}
$$

where $\lambda$ is the wavelength of the light and $k=2 \pi / \lambda$ its wavenumber. In other words, the amplitudes and phases of the wave at two different depths are related to each other via a 2-D Fresnel transform.

\section{Properties of the Fresnel Transform}

Conventional wavelet bases are built using scaled and dilated versions of a suitable template. For building our new wavelet family, it is thus essential to understand how the Fresnel transform behaves with respect to the key operations in multiresolution wavelet theory; i.e., dilation and translation. In Sections III-A-D, we recall properties of the Fresnel transform that are central to our discourse but are also documented in the optics literature [6, pp. 114-119]. In Section III-E, we give a new result which is an uncertainty relation for the Fresnel transform. For clarity, the results are presented for 1-D functions but, using the separability property, they can easily be extended to 2-D functions.

\section{A. Duality}

To compute the inverse of the Fresnel transform we can use following dual relation:

$$
f^{*}(x)=\left(\left(\tilde{f}_{\tau}\right)^{*}\right)_{\tau}^{\sim}(x), \quad f \in L_{2}(\mathbb{R}) .
$$

Computing the inverse Fresnel transform of a function is therefore equivalent to taking its complex conjugate, computing the 
Fresnel transform and again taking the complex conjugate. In other words, the operator $f \mapsto\left(\tilde{f}_{\tau}\right)^{*}$ is involutive.

\section{B. Translation}

As the Fresnel transform is a convolution operator, it is obviously shift-invariant

$$
\left(f\left(\cdot-x_{0}\right)\right)_{\tau}^{\sim}(x)=\tilde{f}_{\tau}\left(x-x_{0}\right), \quad x_{0} \in \mathbb{R} .
$$

\section{Dilation}

The Fresnel transform with parameter $\tau$ of the dilated function $f(x / s)$ is

$$
\left(f\left(\frac{\dot{-}}{s}\right)\right)_{\tau}^{\sim}(x)=\tilde{f}_{\tau / s}\left(\frac{x}{s}\right), \quad s \in \mathbb{R}_{+}^{*} .
$$

This relation involves a dilation by $s$ of the Fresnel transform of $f$ with a rescaled parameter $\tau^{\prime}=\tau / s$. This ratio also appears in the definition of the so-called Fresnel number $N_{F}=(s / \tau)^{2}$, where $\tau^{2}=\lambda d$; it is used to characterize the diffraction of light by a square aperture of halfwidth $s$ and at a distance $d$ [6].

\section{Link With the Fourier Transform}

So far, we have considered the Fresnel transform as a convolution operator. Interestingly, there is also a direct multiplicative relation with the Fourier transform [6]. Computing the Fresnel transform $\tilde{g}_{\tau}$ of a function $g \in L_{2}(\mathbb{R})$ can be done by computing the Fourier transform of an associated function $f(x)=$ $\tau k_{\tau}(x) g(x)$. The frequency variable is then interpreted as an appropriately scaled space variable

$$
\tilde{g}_{\tau}(x)=k_{\tau}(x) \hat{f}\left(\frac{x}{\tau^{2}}\right) .
$$

\section{E. Localization Issues}

Our approach for the construction of a Fresnelet basis will take a wavelet basis and transform it. This still leaves many possibilities to choose the original basis. A suitable basis should take into account one of the least intuitive aspects of holography, namely that the propagation process tends to spread out features that are initially well localized in the object domain. Getting a better understanding of the notion of resolution in holography and setting up a criterion that will guide us in the choice of an optimal wavelet is what we are after in this section.

The tight link between the Fresnel and the Fourier transform (10) suggests that they should both have similar (de)localization properties. Here we derive an uncertainty relation for the Fresnel transform that is the analog of the Heisenberg inequality for the Fourier transform.

In the sequel, we denote the average $\mu_{f}$ of the squared modulus of a function $f \in L_{2}(\mathbb{R})$ by

$$
\mu_{f}=\frac{1}{\|f\|^{2}} \int_{-\infty}^{\infty} x|f(x)|^{2} d x
$$

and its variance $\sigma_{f}^{2}$ around this average by

$$
\sigma_{f}^{2}=\frac{1}{\|f\|^{2}} \int_{-\infty}^{\infty}\left(x-\mu_{f}\right)^{2}|f(x)|^{2} d x .
$$

Theorem 1 (Uncertainty Relation for the Fresnel Transform): Let $g \in L_{2}(\mathbb{R})$ and $\tilde{g}_{\tau} \in L_{2}(\mathbb{R})$ its Fresnel transform with parameter $\tau$. We have following inequality for the product of their variances:

$$
\sigma_{g}^{2} \sigma_{\tilde{g}_{\tau}}^{2} \geq \frac{\tau^{4}}{16 \pi^{2}} .
$$

This inequality is an equality if and only if there exist $x_{0}, \omega_{0}, b$ real and a complex amplitude $a$ such that

$$
g(x)=a e^{i \omega_{0} x} e^{-b\left(x-x_{0}\right)^{2}} e^{-i \pi(x / \tau)^{2}} .
$$

Furthermore, if $g(x)$ is real valued, the following relation holds:

$$
\sigma_{g}^{2} \sigma_{\tilde{g}_{\tau}}^{2} \geq \frac{\tau^{4}}{16 \pi^{2}}+\sigma_{g}^{4} .
$$

This inequality is an equality if and only if there exist $x_{0}, a, b$ real, such that

$$
g(x)=a e^{-b\left(x-x_{0}\right)^{2}} .
$$

Also, (13) implies a lower bound on the variance for $\sigma_{\tilde{g}_{\tau}}$ that is independent of $g$

$$
\sigma_{\tilde{g}_{\tau}}^{2} \geq \frac{\tau^{2}}{2 \pi} .
$$

The proof of Theorem 1 is given in Appendix I.

This result implies that narrow functions yield functions with a large energy support when they are transformed. It suggests that Gaussians and Gabor-like functions, modulated with the kernel function as in (12) should be well suited for processing and reconstructing holograms as they minimize the spatial spreading of the energy. This is especially satisfying because it brings two separate aspects of Gabor's research together: he is both the inventor of holography [5] and of the Gabor transform [11], [12], which is a signal representation as a linear combination of atoms of the form (12). We are not aware of anyone having pointed out this connection before.

We will base our Fresnelets construction on wavelet bases that are close to these optimal functions. Practically, in the case of a digital hologram measurement where a transformed function is available over a finite support and with a given sampling step, we may use the above uncertainty relation to get a bound on the maximal resolution to expect when reconstructing the original function.

A direct illustration of the second part of this Theorem can be found in the example of Section II-B; indeed, it can be verified that the product of the variance of the Gaussian and that of its Fresnel transform achieves the lower bound in (13).

\section{FRESNELET BASES}

To construct our new Fresnelet bases, we will apply a Fresnel transform to a wavelet basis. Here, we will explain what happens when we apply the transform to a general Riesz basis of $L_{2}(\Omega)$, where the dimension of the domain $\Omega$ is arbitrary e.g., $\Omega=\mathbb{R}$ or $\mathbb{R}^{2}$. 


\section{A. Fresnel Transform of a Riesz Basis}

Let $\left\{u_{l}\right\}_{l \in \mathbb{Z}}$ be a Riesz basis of $L_{2}(\Omega)$ and $\left\{v_{l}\right\}_{l \in \mathbb{Z}}$ its dual. Then, $\forall f \in L_{2}(\Omega)$, we can write following expansion:

$$
f=\sum_{l} \underbrace{\left\langle f, v_{l}\right\rangle}_{c_{l}} u_{l}=\sum_{l}\left\langle f, u_{l}\right\rangle v_{l} .
$$

Let $\tilde{u}_{l}=U u_{l}$ where $U$ is a unitary operator (e.g., the Fresnel transform). First, it is easy to see that $U$ maps the biorthogonal set $S=\left\{u_{l}, v_{l}\right\}_{l \in \mathbb{Z}}$ into another biorthogonal set $\tilde{S}=$ $\left\{\tilde{u}_{l}, \tilde{v}_{l}\right\}_{l \in \mathbb{Z}}$

$$
\begin{aligned}
\left\langle\tilde{v}_{l}, \tilde{u}_{m}\right\rangle & =\left\langle U v_{l}, U u_{m}\right\rangle \\
& =\langle\underbrace{U U^{\dagger}}_{1} v_{l}, u_{m}\rangle=\delta_{l, m} .
\end{aligned}
$$

Here $U^{\dagger}$ denotes the adjoint of $U$. Let us now show that $\tilde{S}$ is also complete. For the set $S$, we define the sequence

$$
f_{N}=\sum_{l=1}^{N}\left\langle f, v_{l}\right\rangle u_{l}, \quad \forall f \in L_{2}(\Omega)
$$

and have the completeness equation

$$
\lim _{N \rightarrow \infty}\left\|f-f_{N}\right\|^{2}=0 .
$$

Note that the Riesz basis hypothesis ensures that $f_{N} \in L_{2}(\Omega)$. Because $U$ is unitary, we have

$$
\begin{aligned}
\left\langle f, v_{l}\right\rangle & =\left\langle U f, U v_{l}\right\rangle \\
& =\left\langle\tilde{f}, \tilde{v}_{l}\right\rangle
\end{aligned}
$$

and therefore

$$
\left\|f-f_{N}\right\|^{2}=\left\|\tilde{f}-\tilde{f}_{N}\right\|^{2}
$$

which proves that the transformed set $\tilde{S}$ is complete as well.

Similarly, the Parseval relation (17) can also be used to prove that $S$ and $\tilde{S}$ have the same Riesz bounds. The Riesz bounds are the tightest constants $A>0$ and $B<\infty$ that satisfy the Riesz inequality

$$
A\left\|\left\langle v_{l}, f\right\rangle\right\|_{\ell_{2}}^{2} \leq\|f\|_{L_{2}}^{2} \leq B\left\|\left\langle v_{l}, f\right\rangle\right\|_{\ell_{2}}^{2} .
$$

They are the same for the transformed set

$$
A\left\|\left\langle\tilde{v}_{l}, \tilde{f}\right\rangle\right\|_{\ell_{2}}^{2} \leq\|\tilde{f}\|_{L_{2}}^{2} \leq B\left\|\left\langle\tilde{v}_{l}, \tilde{f}\right\rangle\right\|_{\ell_{2}}^{2} .
$$

Thus, we can conclude that the Fresnel transform, which is a unitary operator from $L_{2}(\Omega)$ into $L_{2}(\Omega)$, maps Riesz bases into other Riesz bases, with the same Riesz bounds. Similarly, if we only consider a subset of basis functions that span a subspace of $L_{2}(\Omega)$ (e.g., a multiresolution subspace) we can show that it maps into a transformed set that is a Riesz basis of the transformed subspace with the same Riesz bounds.

Relation (17) is important for this proof but it is also most relevant for the reconstruction of an image $f$ given its transform $\tilde{f}$. It indicates that we can obtain the expansion coefficients in

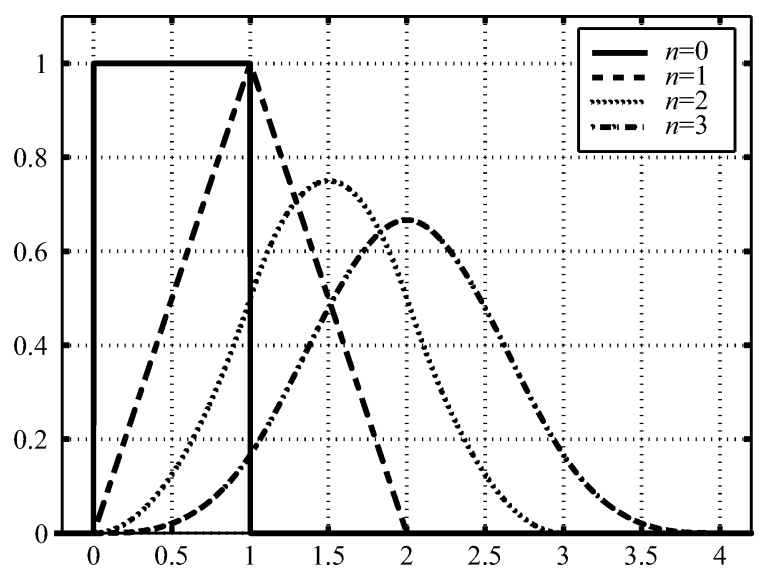

Fig. 1. B-splines of degree $n=0,1,2,3$.

(15) directly by computing the series of inner products $\left\langle\tilde{f}, \tilde{v}_{l}\right\rangle$. This is one of the key ideas for our construction.

\section{B. B-Splines}

The uncertainty relation for the Fresnel transform suggests the use of Gabor-like functions. Unfortunately, these functions cannot yield a multiresolution basis of $L_{2}(\mathbb{R})$. They do not satisfy the partition of unity condition, implying that a representation of a function in term of shifted Gaussians will not converge to the function as the sampling step goes to zero [13]. Furthermore, they do not satisfy a two-scale relation which is required for building wavelets and brings many advantages regarding implementation issues.

We will therefore base our construction on B-splines which are Gaussian-like functions that do yield wavelet bases; they are also well localized in the sense of the uncertainty principle for the Fresnel transform (13).

B-splines [14] are defined in the Fourier domain by

$$
\hat{\beta}^{n}(\nu)=\left(\frac{1-e^{-2 i \pi \nu}}{2 i \pi \nu}\right)^{n+1}=\operatorname{sinc}^{n+1}(\nu) e^{-i \pi \nu(n+1)}
$$

where $\operatorname{sinc}(x)=\sin (\pi x) /(\pi x)$ and $n \in \mathbb{N}$.

The corresponding expression for the B-spline of degree $n$ in the time domain (see Fig. 1) is

$$
\beta^{n}(x)=\Delta^{n+1} * \frac{(x)_{+}^{n}}{n !}
$$

where $(x)_{+}^{n}=\max (0, x)^{n}$ (one-sided power function); $\Delta^{n+1}$ is the $(n+1)$ th finite-difference operator

$$
\Delta^{n+1}=\sum_{k=0}^{n+1}(-1)^{k}\left(\begin{array}{c}
n+1 \\
k
\end{array}\right) \delta(x-k)
$$

which corresponds to the $(n+1)$-fold iteration of the finite difference operator (see [15]): $\Delta=\delta(x)-\delta(x-1)$.

Explicitly, we have following expression for the B-spline of degree $n$ :

$$
\beta^{n}(x)=\sum_{k=0}^{n+1}(-1)^{k}\left(\begin{array}{c}
n+1 \\
k
\end{array}\right) \frac{(x-k)_{+}^{n}}{n !} .
$$


This definition is equivalent to the standard approach where the B-splines of degree $n$ are constructed from the $(n+1)$-fold convolution of a rectangular pulse

$$
\begin{aligned}
& \beta^{n}(x)=\underbrace{\beta^{0} * \cdots * \beta^{0}}_{n+1 \text { times }}(x) \\
& \beta^{0}(x)= \begin{cases}1, & 0<x<1 \\
\frac{1}{2}, & x=0 \text { or } 1 \\
0, & \text { otherwise. }\end{cases}
\end{aligned}
$$

\section{Polynomial Spline Wavelets}

The B-splines satisfy all the requirements of a valid scaling function of $L_{2}(\mathbb{R})$, that is, they satisfy the three necessary and sufficient conditions [8]

$$
\begin{aligned}
& \text { Riesz Basis: } 0<A \leq \sum_{k \in \mathbb{Z}}\left|\hat{\beta}^{n}(\nu+k)\right|^{2} \leq B<\infty \\
& \text { Two-scale relation: } \beta^{n}\left(\frac{x}{2}\right)=\sum_{k \in \mathbb{Z}} h(k) \beta^{n}(x-k) \\
& \text { Partition of unity: } \sum_{k \in \mathbb{Z}} \beta^{n}(x-k)=1
\end{aligned}
$$

where the filter $h(k)$ is the binomial filter $h(k)=$ $1 / 2^{n}\left(\begin{array}{c}n+1 \\ k\end{array}\right)$. These conditions ensure that B-splines can be used to generate a multiresolution analysis of $L_{2}(\mathbb{R})$.

Unser et al. [16] have shown that one can construct a general family of semi-orthogonal spline wavelets of the form

$$
\psi^{n}\left(\frac{x}{2}\right)=\sum_{k} g(k) \beta^{n}(x-k)
$$

such that the functions

$$
\left\{\psi_{j, k}^{n}=2^{(-j / 2)} \psi^{n}\left(2^{-j} x-k\right)\right\}_{j \in \mathbb{Z}, k \in \mathbb{Z}}
$$

form a Riesz basis of $L_{2}(\mathbb{R})$. These wavelets come in different brands: orthogonal, B-spline (of compact support), interpolating, etc... They are all linear combinations of B-splines and are thus entirely specified from the sequence $g(k)$ in (20). Here, we will consider B-spline wavelets [16], which have the shortest support in the family.

The main point here is that by using the properties of the Fresnel transform (linearity, shift invariance and scaling), we can easily derive the family of functions $\left\{\left(\psi_{j, k}^{n}\right)_{\tau}^{\sim}=k_{\tau} * \psi_{j, k}^{n}\right\}_{j \in \mathbb{Z}, k \in \mathbb{Z}}$, provided that we know the Fresnel transform of their main constituent, the B-spline.

\section{Fresnelets}

In this section, we introduce our new wavelets: Fresnelets. They will be specified by taking the Fresnel transform of (20). Thus, the remaining ingredient is to determine the Fresnel transform of the B-splines.

1) F-splines: We define the Fresnel spline, or F-spline of degree $n \in \mathbb{N}$ and parameter $\tau \in \mathbb{R}_{+}^{*}$ (denoted $\tilde{\beta}_{\tau}^{n}(x)$ ) as the Fresnel transform with parameter $\tau$ of a B-spline $\beta^{n}(x)$ of degree $n$

$$
\tilde{\beta}_{\tau}^{n}(x)=\left(\beta^{n} * k_{\tau}\right)(x) .
$$

Theorem 2: The F-spline of degree $n$ and parameter $\tau$ has the closed form

$$
\tilde{\beta}_{\tau}^{n}(x)=\sum_{k=0}^{n+1}(-1)^{k}\left(\begin{array}{c}
n+1 \\
k
\end{array}\right) \frac{u_{n, \tau}(x-k)}{n !}
$$

where

$$
u_{n, \tau}(x)=\int_{0}^{x} \frac{(x-\xi)^{n}}{n !} k_{\tau}(\xi) d \xi .
$$

The proof of Theorem 2 is given in Appendix II.

F-splines have many similarities with B-splines. For example, to get (22), one just substitutes the one-sided power function used in the definition of the B-spline (18) with the functions $u_{n, \tau}$.

Theorem 3: The functions $u_{n, \tau}$ can be calculated recursively

$$
u_{n, \tau}(x)=\frac{\tau}{2 i \pi n !} x^{n-1}-\frac{\tau^{2}}{2 i \pi n} u_{n-2, \tau}(x)+\frac{x}{n} u_{n-1, \tau}(x) .
$$

For $n=0$ we have

$$
u_{0, \tau}(x)=\frac{1}{\sqrt{2}}\left(C\left(\frac{\sqrt{2}}{\tau} x\right)+i S\left(\frac{\sqrt{2}}{\tau} x\right)\right)
$$

where $C(x)$ and $S(x)$ are the so-called Fresnel integrals

$$
C(x)=\int_{0}^{x} \cos \left(\frac{\pi}{2} t^{2}\right) d t, \quad S(x)=\int_{0}^{x} \sin \left(\frac{\pi}{2} t^{2}\right) d t .
$$

For $n=1$ we have

$$
u_{1, \tau}(x)=x u_{0, \tau}(x)-\frac{\tau^{2}}{2 i \pi}\left(k_{\tau}(x)-\frac{1}{\tau}\right) .
$$

The proof of Theorem 3 is given in Appendix III.

This gives us a straightforward way to evaluate the F-splines as the Fresnel integrals can be computed numerically [17]. Furthermore, we can also transpose the well-known B-spline recursion formula

$$
\beta^{n}(x)=\frac{x}{n} \beta^{n-1}(x)+\frac{n+1-x}{n} \beta^{n-1}(x-1)
$$

to the Fresnel domain.

Theorem 4: We have following recursion formula for the F-splines:

$$
\begin{aligned}
& \tilde{\beta}_{\tau}^{n}(x)=\frac{x \tilde{\beta}_{\tau}^{n-1}(x)+(n+1-x) \tilde{\beta}_{\tau}^{n-1}(x-1)}{n+} \\
&+\frac{i \tau^{2}}{2 \pi n} \Delta^{2} \tilde{\beta}_{\tau}^{n-2}(x) .
\end{aligned}
$$

The proof of Theorem 4 is given in Appendix IV.

2) Fresnelet Multiresolutions: Let us now transpose the classical multiresolution relations of wavelet theory to the Fresnelet domain. The two scale relation (19) becomes

$$
\tilde{\beta}_{\tau / 2}^{n}\left(\frac{x}{2}\right)=\sum_{k} h(k) \tilde{\beta}_{\tau}^{n}(x-k) .
$$

In classical wavelet theory, embedded multiresolution spaces are generated through dilation and translation of one single function. The Fresnel transform preserves the embeddedness of those spaces. The important modification comes from the $d i$ lation relation (9) which changes the generating function from one scale to the next. The difference is that in the transformed domain there is one generating function for each scale. 


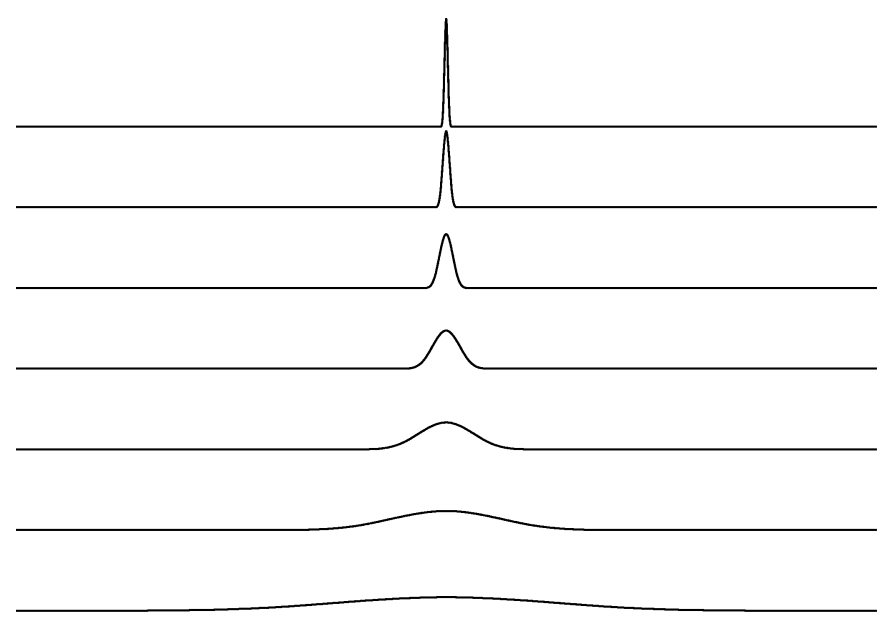

(a)

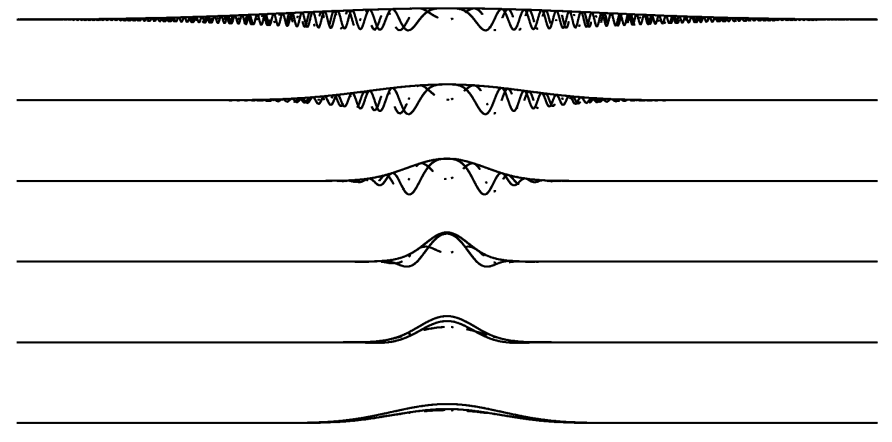

(b)

Fig. 2. B-Spline multiresolution and its Fresnel counterpart. (a) B-splines: $2^{(-j / 2)} \beta^{3}\left(2^{-j} x\right), j=-2,-1,0,1,2,3,4$. (b) Corresponding F-splines: $2^{(-j / 2)} \tilde{\beta}_{\tau 2-j}^{3}\left(2^{-j} x\right)$. In this illustration, $\tau=0.9$. The real part is displayed with a continuous, the imaginary part with a dashed line. For the F-splines, we also show the envelopes of the signals.

Formally, we consider, for $j \in \mathbb{Z}$, the sequence of spaces $\left\{\tilde{V}_{j, \tau}\right\}$ defined as

$$
\tilde{V}_{j, \tau}=\operatorname{span}_{k \in \mathbb{Z}}\left\{\tilde{\beta}_{\tau 2^{-j}}^{n}\left(2^{-j} x-k\right)\right\} \cap L_{2}(\mathbb{R})
$$

corresponding to the sequence of spaces $\left\{V_{j}\right\}$ defined as

$$
V_{j}=\operatorname{span}_{k \in \mathbb{Z}}\left\{\beta^{n}\left(2^{-j} x-k\right)\right\} \cap L_{2}(\mathbb{R}) .
$$

The subspaces $V_{j}$ satisfy the requirements for a multiresolution analysis [8].

1) $V_{j+1} \subset V_{j}$ and $\bigcap V_{j}=0$ and $\overline{U V_{j}}=L_{2}(\mathbb{R})$ (completeness).

2) Scale invariance: $f(x) \in V_{j} \Leftrightarrow f(2 x) \in V_{j-1}$.

3) Shift invariance: $f(x) \in V_{0} \Leftrightarrow f(x-k) \in V_{0}$.

4) Shift-invariant basis: $V_{0}$ has a stable Riesz basis $\left\{\beta^{n}(x-k)\right\}$.

For the sequence $\left\{\tilde{V}_{j, \tau}\right\}$ the shift-invariance is preserved within each scale but requirement 2 is clearly not fulfilled because of the scaling property (9) of the Fresnel transform. We nevertheless get a modified set of multiresolution analysis requirements for the Fresnel transform

$\left.1^{\prime}\right) \tilde{V}_{j+1, \tau} \subset \tilde{V}_{j, \tau}$ and $\cap \tilde{V}_{j, \tau}=0$ and $\bar{U} \tilde{V}_{j, \tau}=L_{2}(\mathbb{R})$ (completeness).

$2^{\prime}$ ) Scale invariance: $f(x) \in \tilde{V}_{j, \tau} \Leftrightarrow \tilde{f}_{\sqrt{3} \tau}(2 x) \in \tilde{V}_{j-1, \tau}$. $\left.3^{\prime}\right)$ Shift invariance: $f(x) \in \tilde{V}_{0, \tau} \Leftrightarrow f(x-k) \in \tilde{V}_{0, \tau}$.

$4^{\prime}$ ) Shift-invariant basis: $V_{0}$ has a stable Riesz basis $\left\{\tilde{\beta}_{\tau}^{n}(x-k)\right\}$.

Condition $2^{\prime}$ is obtained by observing that $f(x) \in \tilde{V}_{j, \tau} \Leftrightarrow f *$ $k_{\tau}^{-1} \in V_{j}$. As we require the $V_{j}$ to satisfy the scale invariance condition 2, we have $f * k_{\tau}^{-1}(2 x) \in V_{j-1}$ hence $\left(f * k_{\tau}^{-1}(2 \cdot)\right) *$ $k_{\tau}(x) \in \tilde{V}_{j-1, \tau}$. And finally

$$
\begin{aligned}
\left(f * k_{\tau}^{-1}(2 \cdot)\right) * k_{\tau}(x) & =\left(f * k_{\tau}^{*}\right) * k_{2 \tau}(2 x) \\
& =e^{i(\pi / 4)} \tilde{f} \tilde{f}_{\sqrt{3} \tau}(2 x) .
\end{aligned}
$$

Specifically, the generating functions corresponding to the B-spline wavelets of (20) are

$$
\tilde{\psi}_{\tau / 2}^{n}\left(\frac{x}{2}\right)=\sum_{k} g(k) \tilde{\beta}_{\tau}^{n}(x-k)
$$

where $\tilde{\beta}_{\tau}^{n}(x)$ is given by (22). The corresponding Fresnelets are such that

$$
\operatorname{span}_{k \in \mathbb{Z}}\left\{\tilde{\psi}_{\tau / 2}^{n}\left(\frac{x}{2}-k\right)\right\} \perp \operatorname{span}_{k \in \mathbb{Z}}\left\{\tilde{\beta}_{\tau / 2}^{n}\left(\frac{x}{2}-k\right)\right\} .
$$

For the multiresolution subspaces, we have that the residual spaces $\tilde{W}_{j, \tau}$ defined as

$$
\tilde{W}_{j, \tau}=\operatorname{span}_{k \in \mathbb{Z}}\left\{\tilde{\psi}_{\tau 2^{-j}}^{n}\left(2^{-j} x-k\right)\right\}
$$

are such that

$$
\tilde{W}_{j+1, \tau} \perp \tilde{V}_{j+1, \tau}
$$

and

$$
\tilde{W}_{j+1, \tau} \oplus \tilde{V}_{j+1, \tau}=\tilde{V}_{j, \tau} .
$$

The above expressions extend the meaning of multiresolution to the fresnelet domain.

3) Fresnelet Multiresolution Example: In Fig. 2 we show a sequence of dyadic scaled $\mathrm{B}$-splines of degree $n=3$ and their counterpart in the Fresnel domain. The effect of the spreading is clearly visible: as the B-splines get finer $(j=1,2,3,4)$ the corresponding F-splines get larger. In contrast to the Fourier transform, as the B-splines get larger $(j=-1,-2)$, the corresponding F-splines' support does not get smaller than the B-splines'. This behavior is in accordance with relation (13).

The main practical consequence for us is: if we want to reconstruct a hologram at a fine scale, that is, express it as a sum of narrow B-splines, the equivalent basis functions on the hologram get larger. Our special choice of Fresnelet bases limits this phenomenon as much as possible; it is nearly optimal in the sense of our uncertainty relation for real functions (13) as they asymptotically converge to Gabor functions [10]. 


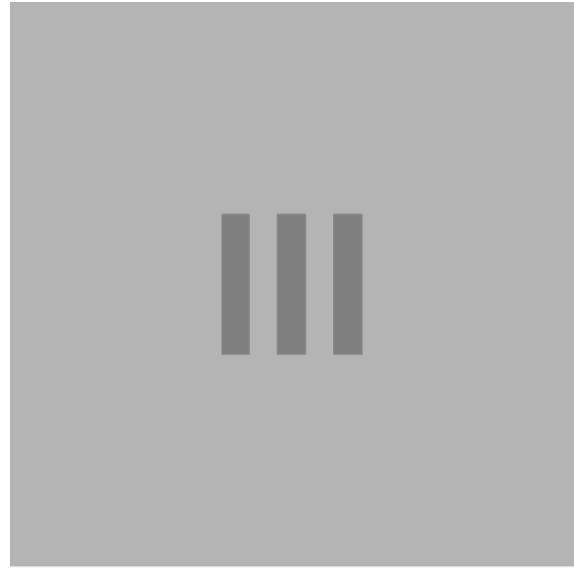

(a)

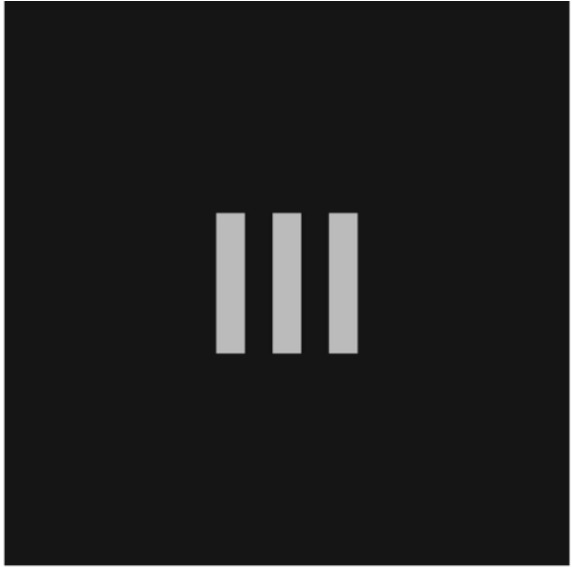

(b)

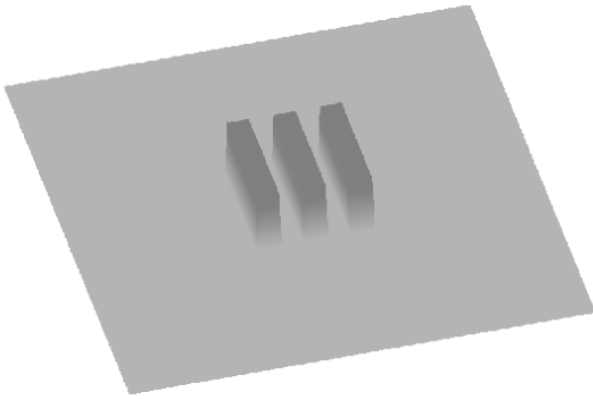

(c)

Fig. 3. (a) Amplitude and (b) phase of the test target. The bars width is $256 \mu \mathrm{m}$. The sampling step is $T=10 \mu \mathrm{m}$ and $512 \times 512$ samples are evaluated. The amplitude is equal to 1 (dark grey) or $\sqrt{2}$ (light grey). The phase is equal to 0 (black) or $\pi / 4$ (light grey). (c) Perspective view. The grayscale is representative for the amplitude and the elevation for the phase.

\section{IMPLEMENTATION OF THE FRESNELET TRANSFORM}

In this section we derive a numerical Fresnelet transform algorithm based on our Fresnelets decomposition.

We consider a function $\tilde{f}_{\tau}(x)$ which is the Fresnel transform of a function $f \in L_{2}(\mathbb{R})$, i.e., $\tilde{f}_{\tau}(x)=k_{\tau} * f(x)$. In a digital holography experiment, this would be the measured phase and amplitude of a propagated wave (without interference with a reference wave). Given some measurements of $\tilde{f}$, the goal is thus to find the best approximation of $f$ in our multiresolution basis. For instance, one can start the process by determining the coefficients $c_{k}$ that give the closest approximation of $f$ (in the $L_{2}$ sense) at the finest scale of representation

$$
f=\sum_{k} c_{k} u(x-k), \quad c_{k}=\langle f, v(x-k)\rangle=\langle\tilde{f}, \tilde{v}(x-k)\rangle
$$

where $u$ and $v$ (respectively $\tilde{u}$ and $\tilde{v}$ ) are dual bases that are linear combinations of B-splines $\beta^{n}$ (respectively F-splines $\tilde{\beta}_{\tau}$ ).

Therefore we only need to compute the inner-products of the transformed function with the shifted F-splines that have been appropriately rescaled

$$
d_{k}=\left\langle f, \frac{1}{h} \beta^{n}\left(\frac{\dot{h}}{h}-k\right)\right\rangle=\left\langle\tilde{f}_{\tau}, \frac{1}{h} \tilde{\beta}_{\tau / h}^{n}\left(\frac{\dot{h}}{h}-k\right)\right\rangle .
$$

Our present implementation is based on a convolution evaluated in the Fourier domain using FFTs. It can be justified as follows. Using Plancherel's identity for the Fourier transform, we express the inner products (28) as

$$
\begin{aligned}
d_{k} & =\left\langle\hat{\tilde{f}}_{\tau}, \hat{\tilde{\beta}}_{\tau / h}^{n}(h \cdot) e^{-2 i \pi k h \cdot}\right\rangle \\
& =\int \hat{\tilde{f}}_{\tau}(\nu) \hat{\tilde{\beta}}_{\tau / h}^{n}(h \nu) e^{-2 i \pi k h \nu} d \nu .
\end{aligned}
$$

In practice, we do not know $\tilde{f}_{\tau}(x)$ in a continuous fashion, but we can easily compute a sampled version of its Fourier transform by applying the FFT to the measured values. If we also approximate the above integral by a Riemann sum, we end up with the implementation formula

$$
d_{k}=\frac{1}{N T} \sum_{l=-N / 2+1}^{N / 2} \hat{\tilde{f}}_{\tau}\left(\frac{l}{N T}\right) \hat{\tilde{\beta}}_{\tau / h}^{n}\left(h \frac{l}{N T}\right) e^{-2 i \pi k h l /(N T)}
$$

where $T$ is the sampling step of the measured function. We can make use of the FFT a second time to compute this sum if we consider sampling steps on the reconstruction side that are multiples of the sampling step of the measured function: $h=m T$, $m=1,2, \ldots$, then

$$
d_{k}=\frac{1}{N T} \sum_{l=-N / 2+1}^{N / 2} \hat{\tilde{f}}_{\tau}\left(\frac{l}{N T}\right) \hat{\tilde{\beta}}_{\tau /(m T)}^{n}\left(m \frac{l}{N}\right) e^{-2 i \pi m(k l / N)} .
$$

The algorithm is thus equivalent to a filtering followed by downsampling by $m$. It is also possible to proceed hierarchically by applying the standard wavelet decomposition algorithm once we have the fine scale coefficients $d_{k}$.

\section{APPLICATIONS AND EXPERIMENTS}

We will now validate our multiresolution Fresnelet-based algorithm and illustrate it in practice on experimental digital holographic data.

\section{A. Simulation: Propagation of a Test Wave Front}

First, we will use our Fresnelet formalism to compute the Fresnel transform of a test pattern that will be used as gold standard to evaluate our algorithm. Although our methodology is more general, for explanatory purposes we consider the case of a plane wave that is being reflected on a test target. The test target is given by three bars. They are of a given thickness and have a different reflectivity than the background they lie on. A plane wave that travels in a normal direction to the target is reflected. In a plane close to the target, the reflected wave's phase is directly proportional to the target's topology whereas the wave's amplitude characterizes the target's reflectivity. The key motif 


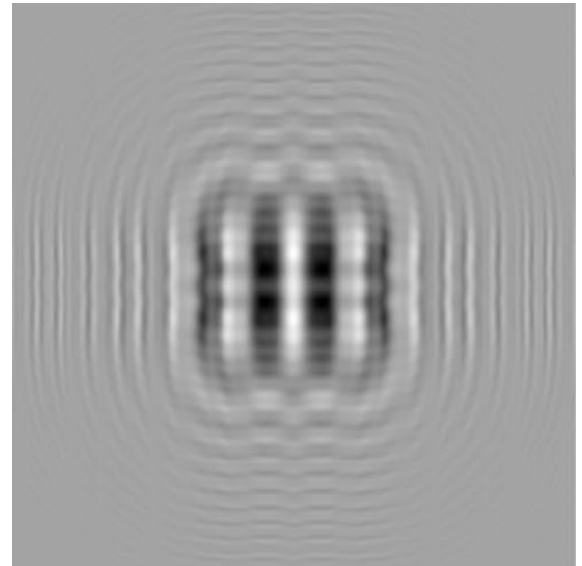

(a)

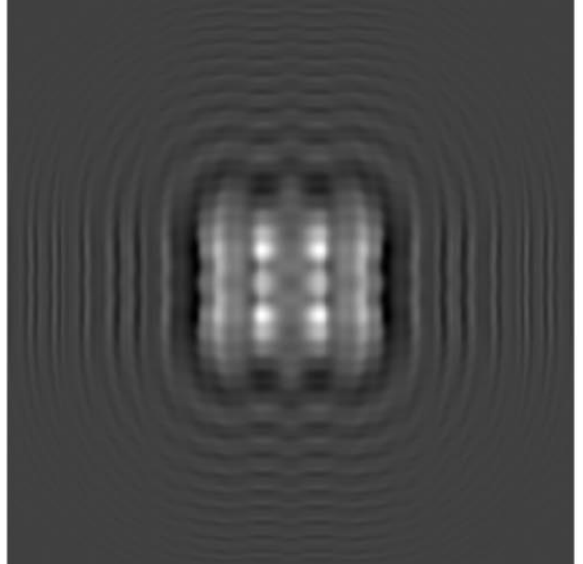

(b)

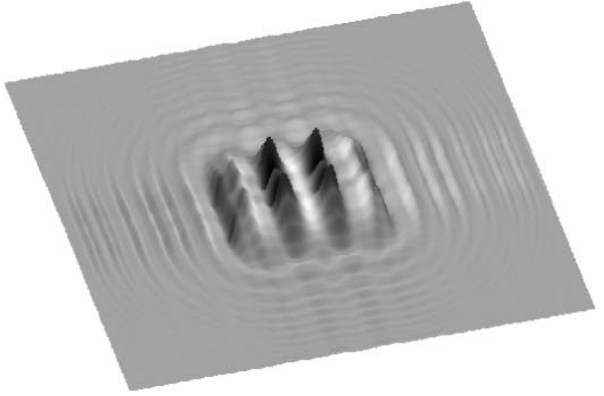

(c)

Fig. 4. Propagated target's (a) amplitude and (b) phase. $d=30 \mathrm{~cm}$ and $\lambda=632.8 \mathrm{~nm}$. Sampling step $T=10 \mu \mathrm{m}$. The sampling step is $T=10 \mu \mathrm{m}$ and $512 \times 512$ samples are evaluated. (c) Perspective view.

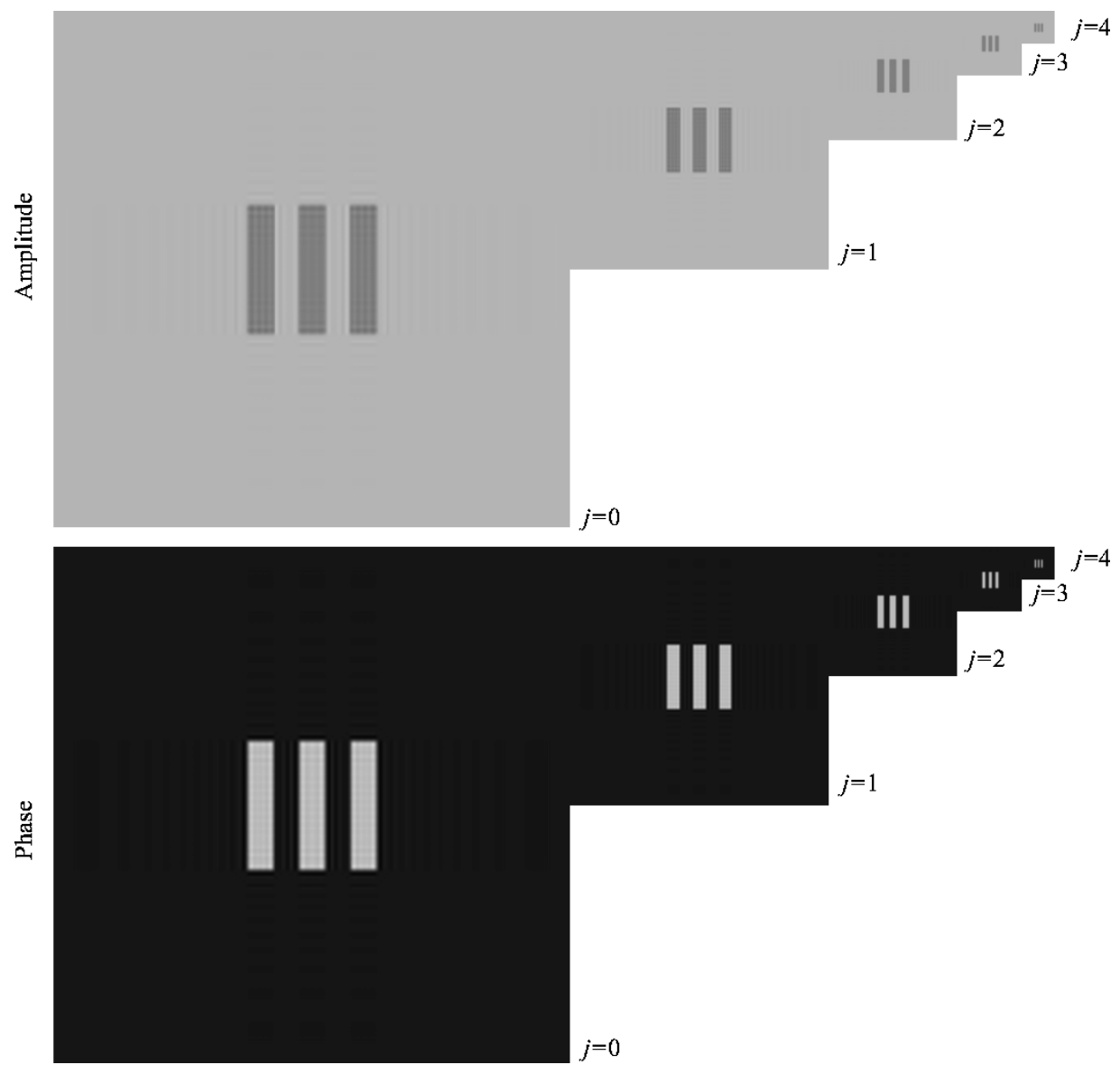

Fig. 5. Reconstructed amplitude (top) and phase (bottom).

of this test pattern is a bar $b(x, y)$ expressed as a tensor product of two B-splines of degree 0

$$
\begin{aligned}
b(x, y) & =e^{i 3 \pi / 4} \beta^{0}\left(\frac{x}{w_{x}}\right) \beta^{0}\left(\frac{y}{w_{y}}\right)+\sqrt{2} \\
& = \begin{cases}e^{i \pi / 4}, & \text { on the bar } \\
\sqrt{2}, & \text { outside. }\end{cases}
\end{aligned}
$$

\section{Its Fresnel transform of parameter $\tau$ is}

$$
\tilde{b}_{\tau}(x, y)=e^{i 3 \pi / 4} \tilde{\beta}_{\tau / w_{x}}^{0}\left(\frac{x}{w_{x}}\right) \tilde{\beta}_{\tau / w_{y}}^{0}\left(\frac{y}{w_{y}}\right)+\sqrt{2} e^{i \pi / 2} .
$$

The amplitude and phase of the target and of the propagated target are shown in Figs. 3 and 4. More complex targets or different phases and amplitudes can be implemented easily with this method. 


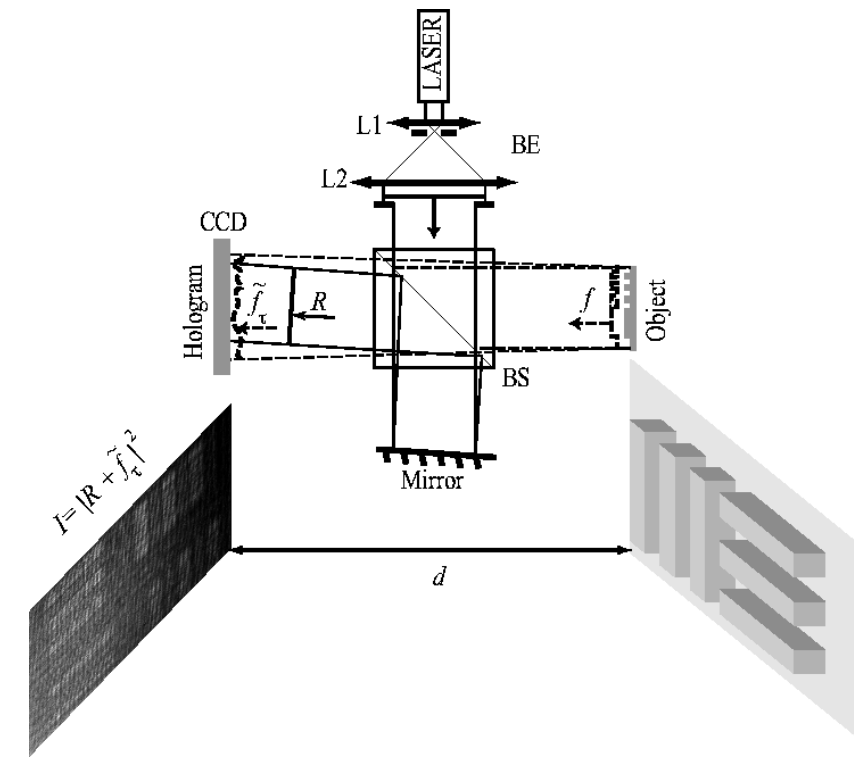

Fig. 6. Experimental digital holography setup. A He-Ne LASER $(\lambda=632.8 \mathrm{~nm})$ beam is expanded by the beam expander (BE) system made of two lenses L1 and L2 and diaphragms. The beam-splitter BS splits the beam. One part illuminates the object. The reflected wave $f$ propagates to the CCD camera at a distance $d$ of the object. In the camera plane the propagated wave is $\tilde{f}_{\tau}$ where $\tau^{2}=\lambda d$. The second part of the beam is reflected by a slightly tilted mirror and impinges on the CCD with a certain angle i.e., its wave vector $\vec{k}=\left(k_{x}, k_{y}, k_{z}\right)$ has nonzero components $k_{x}$ and $k_{y}$. The plane reference wave evaluated in the plane of the CCD is $R(x, y)=A e^{i\left(k_{x} x+k_{y} y\right)}$. The interference of $\tilde{f}_{\tau}$ and $R$ gives the hologram $I=\left|R+\tilde{f}_{\tau}\right|^{2}$.

\section{B. Backpropagation of a Diffracted Complex Wave}

In this experiment, we took the analytical propagated target we just described as the input for our multiresolution Fresnelet transform algorithm. We reconstructed the original target at dyadic scales. In concrete terms, we computed the inner products with F-splines of varying widths: $\tilde{\beta}_{\tau / 2^{j}}^{n}\left(x / 2^{j}\right) \tilde{\beta}_{\tau / 2^{j}}^{n}\left(y / 2^{j}\right)$, $j=0,1,2,3,4, n=3$. We then reconstructed the corresponding images using the underlying spline model. This is also equivalent to running the inverse wavelet transform algorithm up to a specified scale. The results are presented in Fig. 5. At the finest scale $(j=0)$, the sampling step is the same as the one used to sample the propagated wave. To ensure that the reconstructed wave agrees with the initial analytical target, we computed the peak signal to noise ratio (PSNR) of the reconstructed amplitude and phase for the finest reconstruction scale $j=0$. We took following definition of the PSNR:

$$
\text { PSNR }=10 \log _{10}\left(\frac{(\max \{|f|\}-\min \{|f|\})^{2}}{\frac{1}{N_{x} N_{y}} \sum_{k, l}\left|f(k, l)-f^{\prime}(k, l)\right|^{2}}\right)
$$

were $f$ is our (complex) gold standard target and $f^{\prime}$ the reconstructed target. We obtain a PSNR of $23.10 \mathrm{~dB}$. We can thus say that our algorithm reconstructs the target reasonably well.

\section{Hologram Reconstruction}

For this experiment, we considered true holographic data, recorded using a similar system as in [4]. We give a simplified diagram of the experimental setup in Fig. 6.

An object (USAF target) was illuminated using a $\mathrm{He}-\mathrm{Ne}$ laser $(\lambda=632.8 \mathrm{~nm})$. The reflected wave was then directed to the

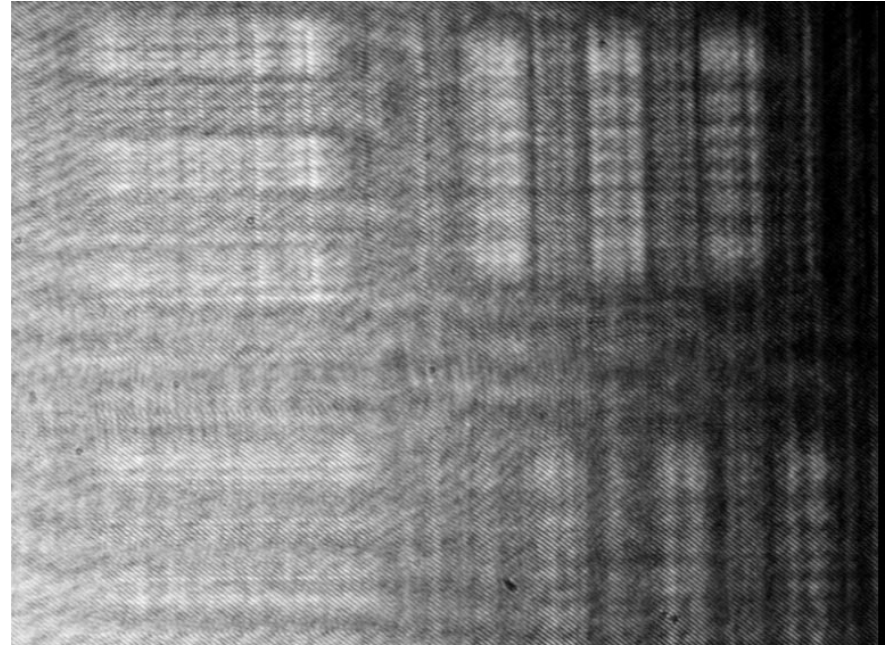

Fig. 7. Measured hologram. There are $776 \times 572$ samples. The sampling step is $10 \mu \mathrm{m}$. (Data courtesy of T. Colomb, F. Montfort and C. Depeursinge, IOA/EPFL.)

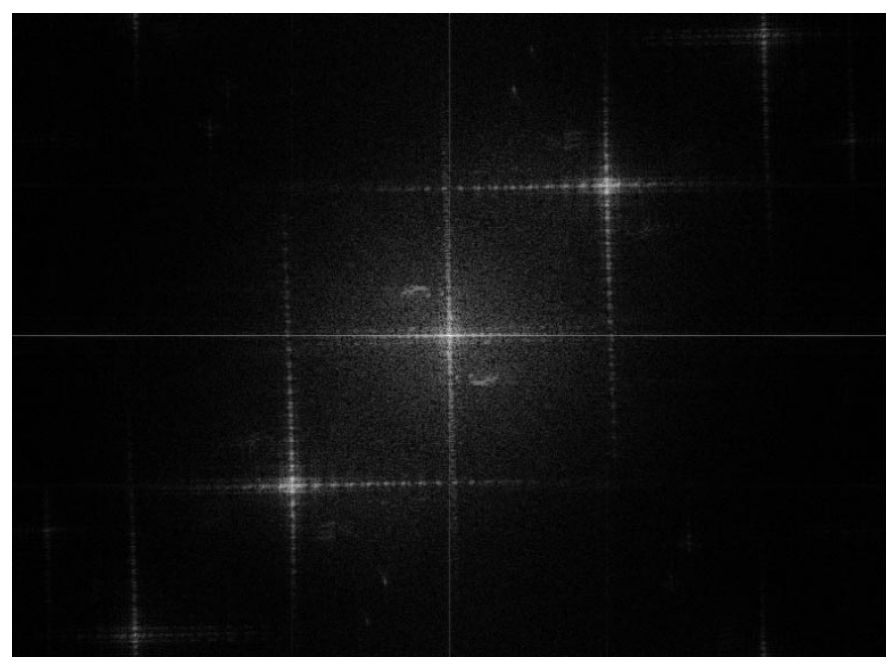

Fig. 8. Absolute value of the Fourier transform of the hologram. The frequency origin is in the center.

$776 \times 572$ pixels CCD camera. The camera recorded the interference (hologram) of this propagated wave with a plane reference wave in an off-axis geometry. The sampling step of the CCD was $T=10 \mu \mathrm{m}$.

We denote $f(x, y)$ the reflected wave in the vicinity of the object and $\tilde{f}_{\tau}(x, y)$ the complex amplitude of the propagated wave in the CCD plane. The hologram is the intensity $I(x, y)$ measured by the camera and results from the interference of the propagated wave $\tilde{f}_{\tau}$ and the reference (plane) wave $R(x, y)=$ $A e^{i\left(k_{x} x+k_{y} y\right)}$

$I(x, y)=\left|\tilde{f}_{\tau}(x, y)+R(x, y)\right|^{2}=\left|\tilde{f}_{\tau}\right|^{2}+|R|^{2}+R^{*} \tilde{f}_{\tau}+R\left(\tilde{f}_{\tau}\right)^{*}$.

The measured hologram is reproduced in Fig. 7.

The two first terms in (31) are known as the zero-order, the third and fourth terms as the image and twin image terms respectively [6]. In the frequency domain, their energy is concentrated around three frequencies: $(0,0)$ for the zero-order, $\left(-k_{x},-k_{y}\right)$ for the image and $\left(k_{x}, k_{y}\right)$ for the twin image. This is clearly visible in Fig. 8. 

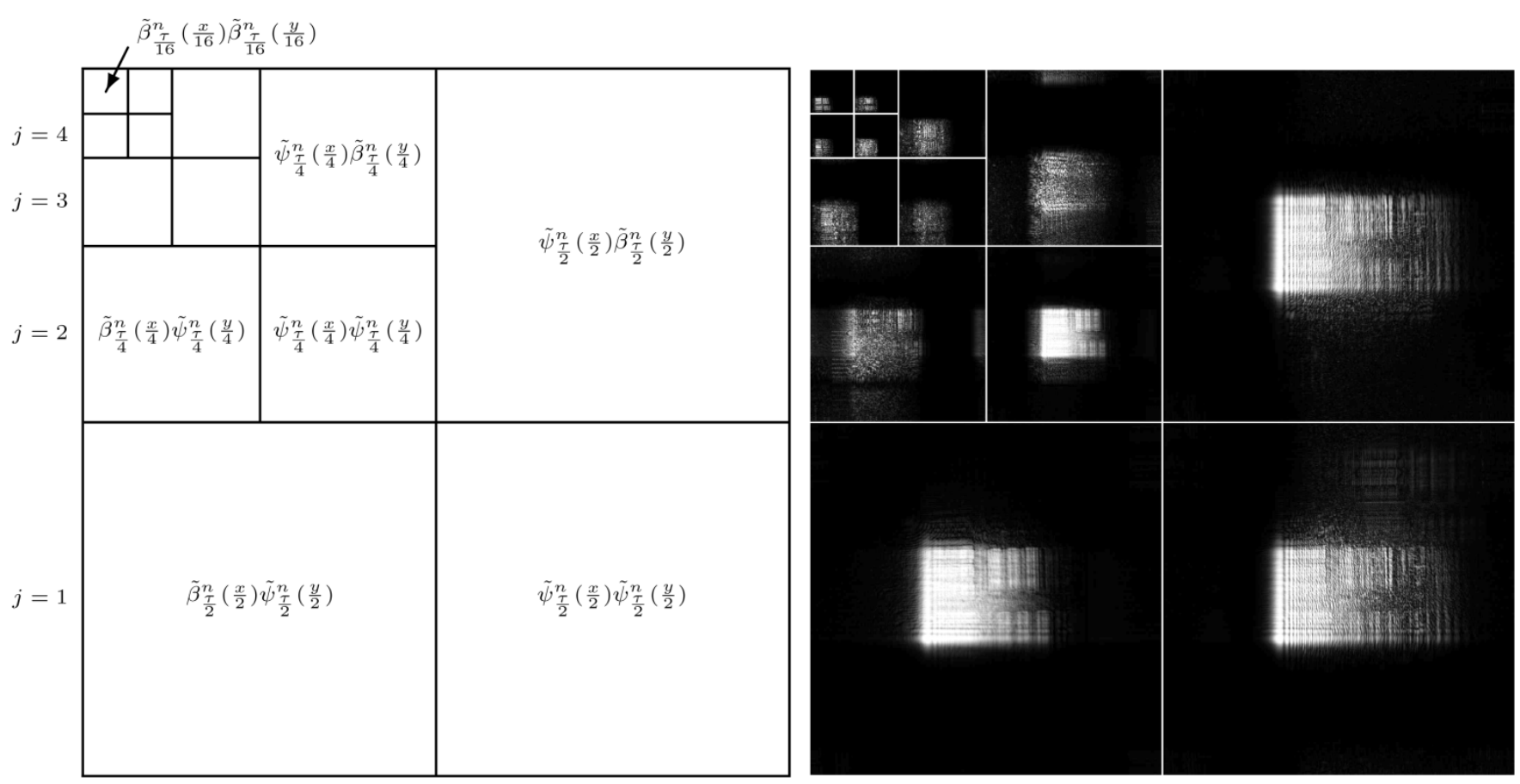

Fig. 9. Fresnelet transform of the modulated hologram $R^{\prime} I$ (coefficient's amplitude). There are $2048 \times 2048$ coefficients.

Prior to reconstructing $f(x, y)$ we multiplied the hologram by a numerical reference wave $R^{\prime}=e^{i\left(k_{x}^{\prime} x+k_{y}^{\prime} y\right)}$

$$
R^{\prime} I=R^{\prime}\left|\tilde{f}_{\tau}\right|^{2}+R^{\prime}|R|^{2}+R^{\prime} R^{*} \tilde{f}_{\tau}+R^{\prime}\left(\tilde{f}_{\tau}\right)^{*} R
$$

The values $k_{x}^{\prime}$ and $k_{y}^{\prime}$ were adjusted precisely to the experimental values $k_{x}, k_{y}$, such that the third term (which is the one we are interested in) becomes $R^{\prime} R^{*} \tilde{f}_{\tau}=a \tilde{f}_{\tau}$ where $a$ is some complex constant. We applied zero padding to the hologram (resulting in a $2048 \times 2048$ input image) to ensure a clear spatial separation of the three reconstructed terms.

We then applied our Fresnelet transform to this (de-)modulated hologram $R^{\prime} I$. The reconstruction distance $d$ was adjusted to $35 \mathrm{~cm}$ resulting in the proper parameter $\tau=\sqrt{\lambda d}$. In Fig. 9 we show the Fresnelet coefficients corresponding to the inner products of $R^{\prime} I$ with the tensor product basis functions $\tilde{\psi}_{\tau / 2^{j}}^{n}\left(x / 2^{j}\right) \tilde{\beta}_{\tau / 2^{j}}^{n}\left(y / 2^{j}\right), \tilde{\psi}_{\tau / 2^{j}}^{n}\left(x / 2^{j}\right) \tilde{\psi}_{\tau / 2^{j}}^{n}\left(y / 2^{j}\right)$, $\tilde{\beta}_{\tau / 2^{j}}^{n}\left(x / 2^{j}\right) \tilde{\psi}_{\tau / 2^{j}}^{n}\left(y / 2^{j}\right)$ and $\tilde{\beta}_{\tau / 2^{J}}^{n}\left(x / 2^{J}\right) \tilde{\beta}_{\tau / 2^{J}}^{n}\left(y / 2^{J}\right)$ for $n=3, j=0, \ldots, J$ and $J=4$. These coefficients are complex and we are only showing their modulus. From these coefficients we could recover the reconstructed signal (amplitude and phase) at any dyadic scale as it is shown in the pyramids of Fig. 10. It is important to remember that all the information to get a finer scale from the coarsest scale (top left) is contained in the subbands of the Fresnelet transform of Fig. 9.

The experiment shows that the three hologram terms are spatially separated in the reconstruction: the zero-order term in the center, the image below left and the twin image up right (not visible). One can also notice how the zero-order term vanishes as the reconstruction scale gets coarser. This is visible in both the pyramid (Fig. 10) where more and more energy goes into the image term as the image gets coarser, and in the
Fresnelet transform (Fig. 9) where the zero-order term coefficient's energy is mainly in the highpass subbands. The explanation for this behavior is the following. As mentioned earlier, the hologram's energy is concentrated around the three frequencies $\left(-k_{x},-k_{y}\right),(0,0)$, and $\left(k_{x}, k_{y}\right)$, corresponding respectively to the image, the zero-order, and the twin image. When we multiply the hologram by $R^{\prime}(x, y) \approx R(x, y)=e^{i\left(k_{x} x+k_{y} y\right)}$, the different terms are shifted by $\left(k_{x}, k_{y}\right)$ in frequency and their new respective locations are $(0,0),\left(k_{x}, k_{y}\right)$ and $\left(2 k_{x}, 2 k_{y}\right)$. As the energy corresponding to the zero order and twin image terms is shifted to high frequencies, it is mainly encoded in the fine scale (highpass) Fresnelet coefficients. Coarse scale reconstructions (which discard the high frequency information) will therefore essentially suppress the zero order or twin image terms, which is a nice feature of our algorithm.

\section{DISCUSSION}

We have seen that the wavefronts reconstructed with the Fresnelet transform from the simulated data agree with the theoretical gold standard and that the algorithm can be applied successfully to reconstruct real-world holographic data as well. Although ringing artifacts may be distinguished at fine scales, they tend to disappear as the scale gets coarser.

The presented method differs from the traditional reconstruction algorithms used in digital holography which implement an inverse Fresnel transform of the data. The Fresnel transform algorithms fall into two main classes [18]. The first approach [Fig. 11(a)], as described in [18], uses the convolution relation (1). It is implemented in the Fourier domain and needs two FFT's. The transformed function's sampling step $T^{\prime}$ is the same as that of the original function. The three terms - the image, the twin image (that is suppressed at all 


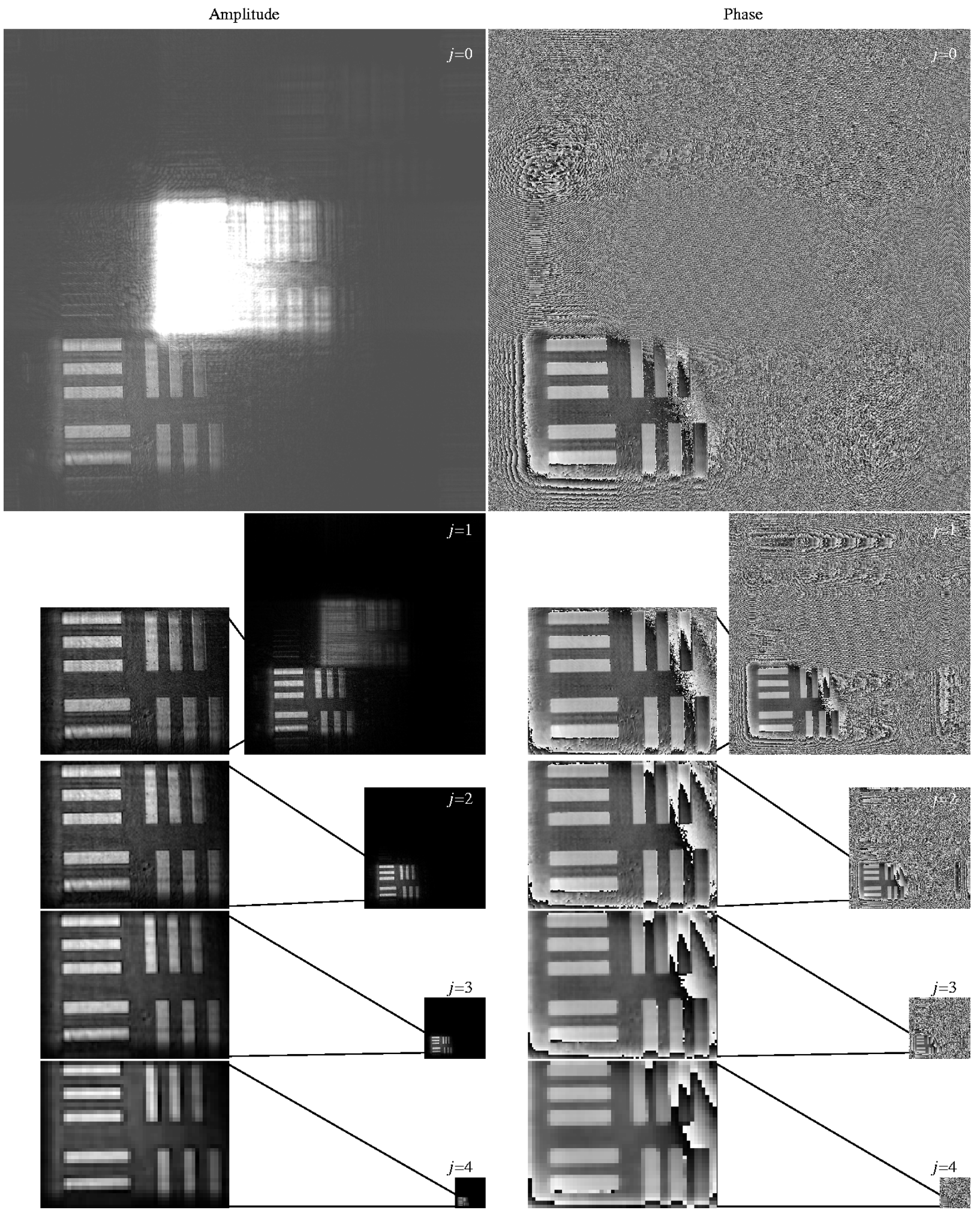

Fig. 10. Reconstructed amplitude and phase from the Fresnelet coefficients in Fig. 9 for $j=0,1,2,3,4$. The contrast was stretched for each image to the full grayscale range, except for the amplitude at $j=0$. At the finest scale $(j=0)$ the size of the images is $2048 \times 2048$.

scales in the Fresnelet algorithm) and the zero-order)—are visible in Fig. 11(a). The second method [Fig. 11(b)] uses the link with the Fourier transform (10) [4], [18]. The discretization of this relation requires only one FFT. As this method relies on the special interpretation of the spatial frequency variable as a rescaled space variable, the sampling step of the transformed function is $T^{\prime}=\lambda d /(N T)$ where $N$ is the number of samples in one direction. Therefore it depends on the distance, the wavelength and the number of measured samples. In particular if the number of samples in the $x$ and $y$ directions are not the 


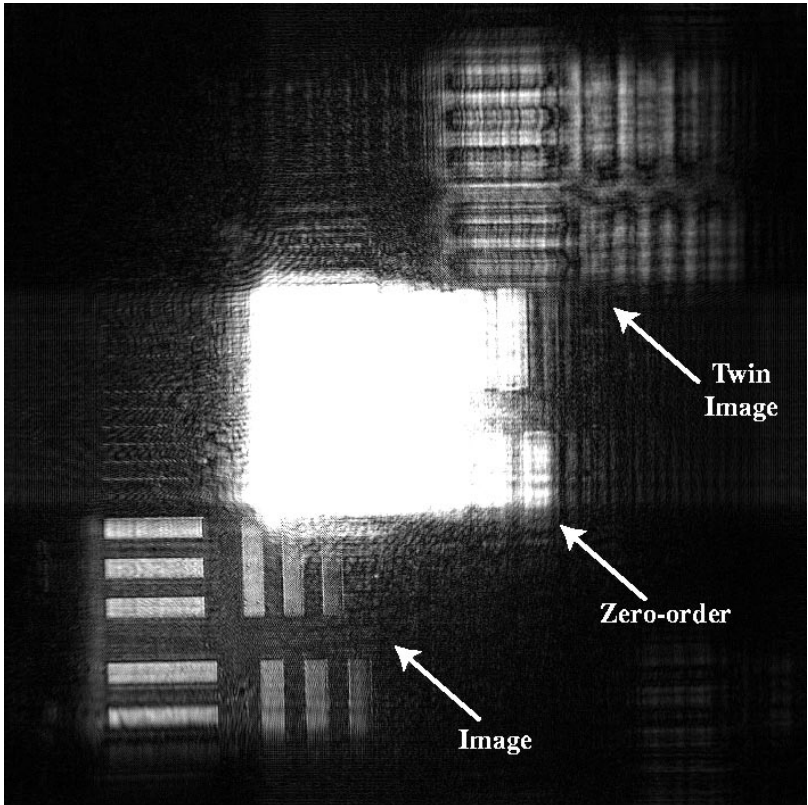

(a)

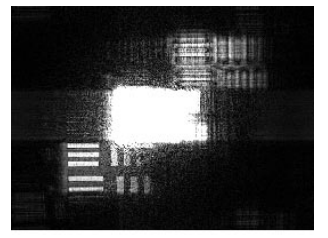

(b)

Fig. 11. Reconstructed amplitudes from the hologram of Fig. 7 using alternative methods based on the discretization of (a) the convolution relation (1) and (b) the Fourier formulation (10) of the Fresnel transform. For (a), the hologram was padded with zeros to a size of $2048 \times 2048$ and the sampling step is $T^{\prime}=T=10 \mu \mathrm{m}$. For the reconstruction in (b) the $776 \times 572$ hologram was fed directly into the algorithm resulting in different sampling steps in the $x$ and $y$ directions: $T_{x}^{\prime}=\lambda d /\left(N_{x} T\right)=28.54 \mu \mathrm{m}$ and $T_{y}^{\prime}=\lambda d /\left(N_{y} T\right)=38.72 \mu \mathrm{m}$.

same, e.g., in Fig. 11(b), the corresponding sampling steps do not agree. In the work of Cuche et al., the parameters are set such that the reconstruction is at approximately one fourth the scale of the digitized hologram.

The first advantage of our approach is that it allows us to choose the sampling step on the reconstruction side. It can be any multiple $T^{\prime}=m T$ for $m=1,2,4,8, \ldots$ The computational cost of our algorithm is the same as that of a filtering in the Fourier domain; i.e., roughly the cost of two FFT's.

Also, as our method is based on the computation of inner products, it leaves more freedom for treating boundary conditions. One possibibility to reduce the influence of the finite support of the CCD camera is to use weighted, or renormalized inner products.

More than just a Fresnel transform, our Fresnelet transform provides us with wavelet coefficients. A remarkable feature is that the energy of the unwanted zero-order and twin images is concentrated within the fine scale subbands. This opens up new perspectives for their selective suppression in the wavelet domain as an alternative to other proposed algorithms ([19], [20]). In addition, it allows us to apply simple wavelet-domain thresholding techniques to reduce the measurement noise in the reconstructed images.

\section{CONCLUSION}

We have constructed a new wavelet basis for the processing and reconstruction of digital holograms by taking advantage of the mathematical properties of the Fresnel transform. We have motivated our choice of B-splines as elementary building blocks based on a new uncertainty relation.

We have demonstrated that the method works and that it is applicable to the reconstruction of real data.

Our method offers several advantages: it allows to reconstruct at different user-specified and wavelength independent scales. Furthermore, reconstructions at coarse scale allow for optimal filtering of the zero-order and the twin image and also result in less noisy images.

\section{APPENDIX I}

\section{A. Proof of Theorem 1}

Proof: We first recall the Heisenberg uncertainty relation for the Fourier transform. Let $f \in L_{2}(\mathbb{R})$. We have the following inequality:

$$
\sigma_{f}^{2} \sigma_{\hat{f}}^{2} \geq \frac{1}{16 \pi^{2}}
$$

This inequality is an equality if and only if there exist $t_{0}, \omega_{0}, b$ real and a complex amplitude $a$ such that

$$
f(t)=a e^{i \omega_{0} t} e^{-b\left(t-t_{0}\right)^{2}}
$$

Let $f(x)=e^{i \pi(x / \tau)^{2}} g(x)$. We start by noting that $f$ and $g$ have the same norm

$$
\|f\|^{2}=\int_{-\infty}^{\infty}\left|e^{i \pi(x / \tau)^{2}} g(x)\right|^{2} d x=\int_{-\infty}^{\infty}|g(x)|^{2} d x=\|g\|^{2}
$$

the same mean

$$
\begin{aligned}
\mu_{f} & =\frac{1}{\|f\|^{2}} \int_{-\infty}^{\infty} x\left|e^{i \pi(x / \tau)^{2}} g(x)\right|^{2} d x \\
& =\frac{1}{\|g\|^{2}} \int_{-\infty}^{\infty} x|g(x)|^{2} d x=\mu_{g}
\end{aligned}
$$

and, finally, the same variances

$$
\begin{aligned}
\sigma_{g}^{2} & =\frac{1}{\|g\|^{2}} \int_{-\infty}^{\infty}\left(x-\mu_{g}\right)^{2}|g(x)|^{2} d x \\
& =\frac{1}{\|g\|^{2}} \int_{-\infty}^{\infty}\left(x-\mu_{g}\right)^{2}\left|e^{i \pi(x / \tau)^{2}} f(x)\right|^{2} d x \\
& =\frac{1}{\|f\|^{2}} \int_{-\infty}^{\infty}\left(x-\mu_{f}\right)^{2}|f(x)|^{2} d x \\
& =\sigma_{f}^{2} .
\end{aligned}
$$

Also, $g$ and $\tilde{g}_{\tau}$ have the same means. Without loss of generality, we will from now on consider that $f$ and $g$ have unit norm $\|f\|=$ $\|g\|=1$ and that they have zero mean $\mu_{f}=\mu_{g}=0$. Using the link between the Fresnel and Fourier transforms (10), we compute the variance of $\tilde{g}$

$$
\begin{aligned}
\sigma_{\tilde{g}_{\tau}}^{2} & =\int_{-\infty}^{\infty} x^{2}\left|\tilde{g}_{\tau}(x)\right|^{2} d x \\
& =\int_{-\infty}^{\infty} x^{2}\left|\frac{1}{\tau} e^{2 i \pi(x / \tau)^{2}} \hat{f}\left(\frac{x}{\tau^{2}}\right)\right|^{2} d x
\end{aligned}
$$




$$
\begin{aligned}
& =\tau^{4} \int_{-\infty}^{\infty} \nu^{2}|\hat{f}(\nu)|^{2} d \nu \\
& =\tau^{4} \sigma_{\hat{f}}^{2} .
\end{aligned}
$$

The product of the variances becomes

$$
\sigma_{g}^{2} \sigma_{\tilde{g}_{\tau}}^{2}=\tau^{4} \sigma_{f}^{2} \sigma_{\hat{f}}^{2} \geq \frac{\tau^{4}}{16 \pi^{2}}
$$

From the Heisenberg uncertainty relation, we know that this inequality is an equality if and only if there exist $x_{0}, \omega_{0}, b$ real and a complex amplitude $a$ such that

$$
g(x)=a e^{i \omega_{0} x} e^{-b\left(x-x_{0}\right)^{2}} e^{-i \pi(x / \tau)^{2}}
$$

To prove the second part of the statement, we compute the variance of the transformed function explicitly

$$
\begin{aligned}
\sigma_{\tilde{g}_{\tau}}^{2} & =\frac{1}{\tau^{2}} \int_{-\infty}^{\infty} x^{2}\left|\hat{f}\left(\frac{x}{\tau^{2}}\right)\right|^{2} d x \\
& =\tau^{4} \int_{-\infty}^{\infty} x^{2}|\hat{f}(x)|^{2} d x \\
& =\frac{\tau^{4}}{4 \pi^{2}} \int_{-\infty}^{\infty}\left|f(x)^{\prime}\right|^{2} d x \\
& =\frac{\tau^{4}}{4 \pi^{2}} \int_{-\infty}^{\infty}\left|g(x)^{\prime}+\frac{2 i \pi x}{\tau^{2}} g(x)\right|^{2} d x
\end{aligned}
$$

If $g(x)$ is real valued, there are no cross terms in the modulus. Thus, we get

$$
\begin{aligned}
\sigma_{\tilde{g}_{\tau}}^{2} & =\tau^{4} \int_{-\infty}^{\infty}|\nu \hat{g}(\nu)|^{2} d \nu+\int_{-\infty}^{\infty}|x g(x)|^{2} d x \\
& =\tau^{4} \sigma_{\hat{g}}^{2}+\sigma_{g}^{2}
\end{aligned}
$$

and finally

$$
\sigma_{\tilde{g}_{\tau}}^{2} \sigma_{g}^{2}=\tau^{4} \sigma_{\hat{g}}^{2} \sigma_{g}^{2}+\sigma_{g}^{4} \geq \frac{\tau^{4}}{16 \pi^{2}}+\sigma_{g}^{4}
$$

which is an equality if and only if there exist $x_{0}, a, b$ real, such that

$$
g(x)=a e^{-b\left(x-x_{0}\right)^{2}} .
$$

To derive the lower bound on the variance $\sigma_{\tilde{g}_{\tau}}^{2}$ we rewrite (13) as

$$
\sigma_{\tilde{g}_{\tau}}^{2} \geq \frac{\tau^{4}}{16 \pi^{2}} \frac{1}{\sigma_{g}^{2}}+\sigma_{g}^{2} .
$$

The right-hand side is minimal for $\sigma_{g}^{2}=\tau^{2} /(4 \pi)$ and therefore

$$
\sigma_{\tilde{g}_{\tau}}^{2} \geq \frac{\tau^{2}}{2 \pi}
$$

\section{APPENDIX II}

\section{A. Proof of Theorem 2}

Proof: $u_{n, \tau}(x)$ satisfies, for $n \geq 1$

$$
\begin{aligned}
u_{n, \tau}^{\prime}(x) & =\frac{d}{d x} \int_{0}^{x} \frac{(x-\xi)^{n}}{n !} k_{\tau}(\xi) d \xi \\
& =\int_{0}^{x} \frac{(x-\xi)^{n-1}}{(n-1) !} k_{\tau}(\xi) d \xi \\
& =u_{n-1, \tau(x)}
\end{aligned}
$$

and for $n=0$

$$
u_{0, \tau}^{\prime}(x)=k_{\tau}(x) .
$$

Therefore, by differentiating $u_{n, \tau}(n+1)$ times, we hit the kernel of the Fresnel Transform operator

$$
u_{n, \tau}^{(n+1)}(x)=k_{\tau}(x) .
$$

We can now calculate the Fresnel transform of a B-spline of degree $n$

$$
\begin{aligned}
\tilde{\beta}_{\tau}^{n}(x) & =\left(\beta^{n} * k_{\tau}\right)(x) \\
& =\left(\beta^{n} * u_{n, \tau}^{(n+1)}\right)(x) .
\end{aligned}
$$

As differentiation and convolution commute, we have

$$
\begin{aligned}
\tilde{\beta}_{\tau}^{n} & =\left(\frac{d^{n+1}}{d x^{n+1}} \beta^{n}\right) * u_{n, \tau}(x) \\
& =\left(\sum_{k=0}^{n+1}(-1)^{k}\left(\begin{array}{c}
n+1 \\
k
\end{array}\right) \frac{\delta(x-k)}{n !}\right) * u_{n, \tau}(x) \\
& =\sum_{k=0}^{n+1}(-1)^{k}\left(\begin{array}{c}
n+1 \\
k
\end{array}\right) \frac{u_{n, \tau}(x-k)}{n !} .
\end{aligned}
$$

\section{APPENDIX III}

\section{A. Proof of Theorem 3}

Proof: We integrate (23) by parts, using $(d / d x) k_{\tau}(x)=$ $\left(2 i \pi x / \tau^{2}\right) k_{\tau}(x)$

$$
\begin{aligned}
u_{n, \tau}(x)= & \int_{0}^{x} \frac{(x-\xi)^{n}}{n !} k_{\tau}(\xi) d \xi \\
= & {\left[-\frac{(x-\xi)^{n+1}}{(n+1) !} k_{\tau}(\xi)\right]_{0}^{x} } \\
& -\int_{0}^{x}-\frac{(x-\xi)^{n+1}}{(n+1) !} \frac{2 i \pi \xi}{\tau^{2}} k_{\tau}(\xi) d \xi \\
= & \frac{x^{n+1}}{\tau(n+1) !}-\frac{2 i \pi}{\tau^{2}} \\
& \times\left(\int_{0}^{x} \frac{(x-\xi)^{n+2}}{(n+1) !} k_{\tau}(\xi) d \xi\right. \\
= & \left.\frac{x^{n+1}}{\tau(n+1) !} \int_{0}^{x} \frac{(x-\xi)^{n+1}}{(n+1) !} k_{\tau}(\xi) d \xi\right) \\
& -\frac{2 i \pi}{\tau^{2}}\left((n+2) u_{n+2, \tau}(x)-x u_{n+1, \tau}(x)\right)
\end{aligned}
$$


which we rewrite under the form (24). The expressions for $u_{0, \tau}(x)$ and $u_{1, \tau}(x)$ follow immediately from the general definitions of $u_{n, \tau}$, the Fresnel integrals and the recursion formula (24).

\section{APPENDIX IV}

\section{A. Proof of Theorem 4}

Proof: We begin by computing the Fresnel transform of a B-spline that is multiplied by $x$

$$
\begin{aligned}
\left(x \beta^{n}\right)_{\tau}^{\sim}(x) & =\int_{-\infty}^{\infty}(x-\xi) \beta^{n}(x-\xi) k_{\tau}(\xi) d \xi \\
& =x \tilde{\beta}_{\tau}^{n}(x)-\int_{-\infty}^{\infty} \frac{\tau^{2}}{2 i \pi} \beta^{n}(x-\xi) \frac{d}{d x} k_{\tau}(\xi) d \xi \\
& =x \tilde{\beta}_{\tau}^{n}(x)-\frac{\tau^{2}}{2 i \pi}\left(\frac{d}{d x} \beta^{n}(x)\right)_{\tau}^{\sim} .
\end{aligned}
$$

We can now use the B-spline's differentiation formula [14]

$$
\frac{d}{d x} \beta^{n}(x)=\beta^{n-1}(x)-\beta^{n-1}(x-1)=\Delta \beta^{n-1}(x)
$$

to get

$$
\left(x \beta^{n}\right)_{\tau}^{\sim}(x)=x \tilde{\beta}_{\tau}^{n}(x)-\frac{\tau^{2}}{2 i \pi} \Delta \tilde{\beta}_{\tau}^{n-1}(x) .
$$

We rewrite (25) as

$$
\beta^{n}(x)=\frac{1}{n} \Delta\left(x \beta^{n-1}(x)\right)+\beta^{n-1}(x-1)
$$

to finally get its Fresnel transform

$$
\begin{aligned}
\tilde{\beta}_{\tau}^{n}(x)= & \frac{1}{n} \Delta\left(x \tilde{\beta}_{\tau}^{n-1}(x)-\frac{\tau^{2}}{2 i \pi} \Delta \tilde{\beta}_{\tau}^{n-2}(x)\right) \\
& +\tilde{\beta}_{\tau}^{n-1}(x-1) \\
= & \frac{x \tilde{\beta}_{\tau}^{n-1}(x)+(n+1-x) \tilde{\beta}_{\tau}^{n-1}(x-1)}{n} \\
& +\frac{i \tau^{2}}{2 \pi n} \Delta^{2} \tilde{\beta}_{\tau}^{n-2}(x) .
\end{aligned}
$$

[4] E. Cuche, F. Bevilacqua, and C. Depeursinge, "Digital holography for quantitative phase-contrast imaging," Opt. Lett., vol. 24, no. 5, pp. 291-293, Mar. 1999.

[5] D. Gabor, "A new microscopic principle," Nature, vol. 161, no. 4098, pp. 777-778, 1948.

[6] J. W. Goodman, Introduction to Fourier Optics, 2nd ed. New York: McGraw-Hill, 1996.

[7] E. Cuche, P. Marquet, and C. Depeursinge, "Simultaneous amplitudecontrast and quantitative phase-constrast microscopy by numerical reconstruction of Fresnel off-axis holograms," Appl. Opt., vol. 38, no. 34, pp. 6994-7001, Dec. 1999.

[8] G. Strang and T. Nguyen, Wavelets and Filter Banks. Wellesley, MA: Wellesley-Cambridge, 1996.

[9] T. Blu and M. Unser, "Quantitative Fourier analysis of approximation techniques: Part II-Wavelets," IEEE Trans. Signal Processing, vol. 47, pp. 2796-2806, Oct. 1999.

[10] M. Unser, A. Aldroubi, and M. Eden, "On the asymptotic convergence of B-spline wavelets to Gabor functions," IEEE Trans. Inform. Theory, vol. 38, pp. 864-872, Mar. 1992.

[11] D. Gabor, "Theory of communication," J. Inst. Elect. Eng. (London), vol. 93, pp. 429-457, 1946.

[12] A. J. E. M. Janssen, "Gabor representation of generalized functions," $J$. Math. Anal. Appl., vol. 83, pp. 377-394, Oct. 1981.

[13] M. Unser, "Sampling-50 years after Shannon," Proc. IEEE, vol. 88, pp. 569-587, Apr. 2000

[14] — "Splines: A perfect fit for signal and image processing," IEEE Signal Processing Mag., vol. 16, no. 6, pp. 22-38, Nov. 1999.

[15] M. Unser and T. Blu, "Fractional splines and wavelets," SIAM Review, vol. 42, no. 1, pp. 43-67, Mar. 2000.

[16] M. Unser, A. Aldroubi, and M. Eden, "A family of polynomial spline wavelet transforms," Signal Processing, vol. 30, no. 2, pp. 141-162, Jan. 1993.

[17] W. H. Press, S. A. Teukolsky, W. T. Vetterling, and B. P. Flannery, Numerical Recipes in $C, 2$ nd ed. Cambridge, U.K: Cambridge Univ. Press, 1992.

[18] T. Kreis, M. Adams, and W. Jüptner, "Methods of digital holography: A comparison," Proc. SPIE, vol. 3098, pp. 224-233, 1997.

[19] E. Cuche, P. Marquet, and C. Depeursinge, "Spatial filtering for zeroorder and twin-image elimination in digital off-axis holography," Appl. Opt., vol. 39, no. 23, pp. 4070-4075, Aug. 2000.

[20] T. Kreis and W. Jüptner, "Suppression of the DC term in digital holography," Opt. Eng., vol. 36, no. 8, pp. 2357-2360, Aug. 1997.

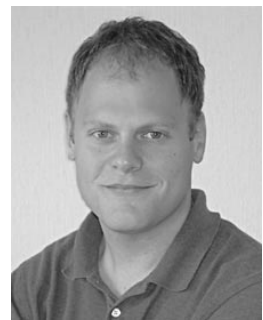

Michael Liebling ( $S^{\prime} 01$ ) was born in Zurich, Switzerland, in 1976. He graduated as a physics engineering M.Sc. from EPFL (École Polytechnique Fédérale de Lausanne), Switzerland, in 2000. He is currently with the Biomedical Imaging Group, EPFL, where he is working toward a Ph.D. degree on digital holography.

His research interests include image processing, inverse problems, optics, and wavelets.

\section{ACKNOWLEDGMENT}

This work is part of the joint project in biomedical engineering of HUG/UNIL/EPFL/UNIGE/HCV: MICRO-DIAG. The authors would like to thank C. Depeursinge, T. Colomb, and F. Montfort for providing the experimental hologram data.

\section{REFERENCES}

[1] J. W. Goodman and R. W. Lawrence, "Digital image formation from electronically detected holograms," Appl. Phys. Lett., vol. 11, no. 3, pp. 77-79, Aug. 1967.

[2] L. P. Yaroslavskii and N. S. Merzlyakov, Methods of Digital Holography. New York: Consultants Bureau, 1980.

[3] U. Schnars and W. Jüptner, "Direct recording of holograms by a CCD target and numerical reconstruction," Appl. Opt., vol. 33, no. 2, pp. 179-181, Jan. 1994.

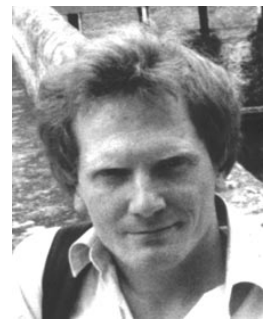

Thierry Blu (M'96) was born in Orléans, France, in 1964. He received the "Diplôme d'ingénieur" from École Polytechnique, France, in 1986 and from Télécom Paris (ENST), France, in 1988. In 1996, he received the Ph.D. in electrical engineering from ENST for a study on iterated rational filterbanks, applied to wideband audio coding.

$\mathrm{He}$ is with the Biomedical Imaging Group at the Swiss Federal Institute of Technology (EPFL), Lausanne, Switzerland, on leave from France Télécom National Center for Telecommunications Studies (CNET), Issy-les- Moulineaux, France. His research interests: (multi)wavelets, multiresolution analysis, multirate filterbanks, approximation and sampling theory, psychoacoustics, optics, wave propagation.

Dr. Blu is currently serving as an Associate Editor for the IEEE TRANSACTIONS ON IMAGE PROCESSING. 


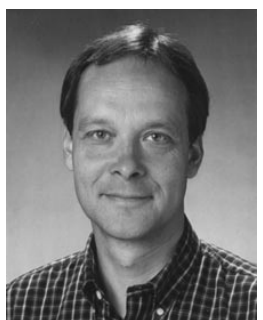

Michael Unser (M'89-SM'94-F'99) received the M.S. (summa cum laude) and Ph.D. degrees in electrical engineering in 1981 and 1984, respectively, from the Swiss Federal Institute of Technology in Lausanne (EPFL), Switzerland.

From 1985 to 1997 , he was with the Biomedical Engineering and Instrumentation Program, National Institutes of Health, Bethesda. He is now Professor and Head of the Biomedical Imaging Group at the EPFL. His main research area is biomedical image processing. He has a strong interest in sampling theories, multiresolution algorithms, wavelets, and the use of splines for image processing. He is the author of 100 published journal papers in these areas. $\mathrm{He}$ is on the editorial board of Signal Processing.

Dr. Unser is Associate Editor-in-Chief for the IEEE TRANSACTIONS ON MEDicAl IMAGING. He is on the editorial boards of several other journals, including IEEE Signal Processing Magazine, IEEE TRANSACTIONS ON IMAge Processing (1992-1995), and IEEE Signal Processing LeTters (1994-1998). He serves as regular chair for the SPIE Conference on Wavelets, held annually since 1993 . He was general co-chair of the first IEEE International Symposium on Biomedical Imaging, held in Washington, DC, in 2002. He received the 1995 Best Paper Award and the 2000 Magazine Award from the IEEE Signal Processing Society. In January 1999, he was elected Fellow of the IEEE with the citation: "for contributions to the theory and practice of splines in signal processing." 